\section{Die Unterkunft}

Eine eigene Unterkunftsmöglichkeit seitens der AHK besteht nicht. Schon von Deutschland aus hatte ich aber über cairoscholars für den ersten Monat eine Unterkunft organisieren können. Ich teilte mir eine WG mit einer deutschen Praktikantin der AHK. Meine Unterkunft für Dezember und Januar bekam ich auch über cairoscholars. Diesmal wohnte ich mit einer Australierin und einer US-Amerikanerin zusammen.

Grundsätzlich sollte man sich potentielle WG-Zimmer vorher anschauen. Der Standard kann erheblich von dem gewohnten deutschen abweichen. Man sollte sich auch überlegen, ob man inmitten von „expats“, also Ausländern, wohnen möchte. Diese Viertel sind tendenziell teurer und das kulinarische und kulturelle Angebot ist auf die Ausländer ausgerichtet. Ich habe es bei meiner zweiten Unterkunft vorgezogen, in einem Einheimischenviertel zu wohnen. So wohnt man nicht in einer westlichen Parallelwelt, sondern kriegt mehr von dem wahren ägyptischen Leben mit.

\section{Kairo}

\section{Die Stadt}

Kairo ist einfach eine faszinierende Stadt. Sie vereint das alte Ägypten auf der einen mit der islamischen Kultur auf der anderen Seite. Sowohl die Pyramiden als auch unzählige alte und prachtvolle Moscheen gehören zum Stadtbild. Wenn man sich darauf einlässt, kann man jeden Tag etwas Neues entdecken. Auch wenn die Stadt in vielerlei Hinsicht modern erscheint, sollte man nie vergessen, dass die Bevölkerung tief religiös ist. Ich bin auch bei $27^{\circ} \mathrm{C}$ mit einem langärmligen Mantel unterwegs gewesen. Ein Kopftuch hatte ich jedoch nur auf, wenn dies in bestimmten Moscheen vorgeschrieben war.

Die Religion ist allgegenwärtig. Selbst meine Büronachbarin, die kein Kopftuch trug und sehr modisch gekleidet war, hat im Büro gebetet. Fünf Mal am Tag hört man - egal, wo man ist - den Ruf der unzähligen Muezzine. Nach einiger Zeit bin ich davon jedoch frühmorgens nicht mehr aufgewacht.

\section{Die Sprache}

Es ist durchaus möglich, ohne Arabischkenntnisse durch den Alltag zu kommen. Je weiter man sich jedoch von den Touristenzentren entfernt, desto mehr ist es zu empfehlen, einige Brocken Ägyptisch-Arabisch zu sprechen. Hocharabisch, wie man es meistens in den deutschen Sprachschulen lernt, taugt nur bedingt. Ich habe schon vor meinem Aufenthalt Sprachunterricht genommen und dann meine Kenntnisse vor Ort mit einer Privatlehrerin vertieft.

\section{Als Frau}

Gefährlich ist es nicht, sich als Frau allein in Kairo zu bewegen. Man sollte aber wissen, dass man als europäisch aussehende Frau bereits aus großer Entfernung auffällt und allein unterwegs eine Besonderheit darstellt. Viele, insbesondere junge, Männer können sich Sprüche, Pfiffe und dergleichen nicht verkneifen. Damit muss man umgehen können. Nach einiger Zeit hatte ich mir ein dickes Fell zugelegt und habe die Männer auf der Straße nicht mehr angeguckt, was ein wenig hilft.

Die Ägypter sind aber ansonsten ein sehr herzliches und hilfsbereites Volk. Gerade auch die älteren Männer haben mich sehr respektvoll behandelt. Arabischkenntnisse helfen einem dabei oft weiter und schaffen Vertrauen.

\section{Fazit}

Ich kann jeden, der mit dem Gedanken spielt, eine Station in Kairo zu verbringen, nur dazu ermutigen, es war einfach eine unvergessliche Zeit. Wer dazu Fragen hat, kann sich gerne an mich wenden.

\title{
LL.M. oder Dr. jur. - eine Frauenfrage?
}

\section{Dr. Anna Katharina Mangold, LL.M. (Cambridge) \\ Wissenschaftliche Mitarbeiterin, Freiburg i. Br.}

Oft machen sich Jurastudentinnen bereits im ersten Semester Gedanken darüber, ob sie einen „LL.M.“ machen sollen. Der LL.M. muss besonders attraktiv erscheinen, wenn selbst Erstsemesterinnen schon von ihm gehört haben und sich von ihm angezogen fühlen. Dem anglo-amerikanischen Universitätssystem entstammend verweist der „Master auf Laws“ mit dem Plural, Magistra der Rechte, auf die frühere Ausbildung sowohl im weltlichen als auch im kanonischen Recht. Inzwischen beschränkt sich das Studium längst auf das weltliche Recht. Das anglo-amerikanische System der universitären Jurist(inn)enausbildung unterscheidet sich deutlich vom deutschen mit seinem Abschlussziel „Erstes Staatsexamen“. So sind in den USA und Großbritannien zunächst lediglich Grundkenntnisse in Jura zu erwerben, die mit einem Bachelor belohnt werden (in England dem LL.B., Bachelor of Laws). Der Meistergrad ist dagegen vor allem für Studierende interessant, die eine ausgesprochen akademische Neigung haben oder auf einem bestimmten Gebiet vertiefte Kenntnisse erwerben wollen.

Für deutsche Studierende kann einer dieser beiden Gründe ausschlaggebend sein, einen LL.M. in Erwägung zu ziehen. Darüber hinaus aber sehen vor allem international tätige Kanzleien und Wirtschaftsunternehmen den Titel gerne, verspricht er doch vertiefte Sprachkenntnisse, insbesondere juristische. Zudem ist das Masterstudium mit einer Dauer von nur einem akademischen Jahr ein zeitlich überschaubares Projekt, das keinen allzu schweren Einschnitt in die übrige berufliche und persönliche Lebensplanung bedeutet. 
Die Attraktivität des LL.M. für Wirtschaftsunternehmen und Kanzleien führt dazu, dass sich an Eliteunis wie Oxbridge oder den amerikanischen Ivy-League-Universities-Einladungen zu Recruiting-Veranstaltungen in den Postfächern der Studierenden stapeln. In Cambridge etwa gaben sich die in Deutschland tätigen Großkanzleien die Klinke in die Hand in ihrem Eifer, das deutsche Kontingent im LL.M. in die feinsten Lokale zu führen. Zur heimlichen Freude der Deutschen durften die Österreicher nicht mit - ein Beleg dafür, dass wohl doch das vielgescholtene, offenbar aber beliebte Staatsexamen entscheidet, durch das sich alle Deutschen zwangsläufig gequält, zugleich aber auch besonders qualifiziert haben.

Die berühmtesten Universitäten in den USA und Großbritannien verlangen für die Zulassung zum LL.M. durchweg ein besseres Prädikatsexamen, bei speziellen Fachrichtungen reicht bisweilen auch eine einschlägige Schwerpunktsetzung im Studium. Finanziert wird der LL.M. entweder durch ein Stipendium - in Deutschland vor allem der Studienstiftung und des DAAD - oder aus eigener Tasche. Gerade ein LL.M. in den USA kann sehr ins Geld gehen, LL.M.-Studiengänge in der EU sind dagegen gedeckelt auf den Heimattarif (der freilich gelegentlich nicht unerheblich sein kann).

Der größte Konkurrent des LL.M. ist der Doktortitel. Eine Doktorarbeit kann von einem halben Jahr bis zu mehreren Jahren dauern, abhängig von Thema, Disziplin und betreuender Person. Festzuhalten ist, dass es so viele Gründe gibt, eine Doktorarbeit zu verfassen, wie Doktorand(inn)en. Ob es wissenschaftliches Interesse, die Neugier auf vertiefende Behandlung eines speziellen Themas, Lust auf selbstbestimmtes Arbeiten oder einfach der Wunsch nach dem Titel ist, grundsätzlich ist kein Grund schlechter als ein anderer. Wichtig ist allein, sich über diesen Grund Rechenschaft abzulegen, denn er sollte ausschlaggebend sein für die Ausgestaltung der Promotion. Eine Juristin, die den Titel aus Prestigegründen anstrebt - was keineswegs zu verurteilen ist! -, sollte möglichst nicht über ein unüberschaubares Thema promovieren; umgekehrt sollte die wissenschaftlich interessierte Promovendin sich nicht an die
Auslegung von Unterabsatz 7 b) einer abgelegenen Vorschrift verschwenden.

Klassischerweise wird der Doktor entweder über eine Lehrstuhlstelle oder ein Stipendium finanziert. Beides hat Vor- und Nachteile. Der Lehrstuhljob gewährt engen Kontakt mit der Betreuungsperson, vermittelt Lehrerfahrung, verlangt andererseits aber Arbeitseinsatz, der nicht notwendigerweise der Doktorarbeit zugutekommt. Demgegenüber lässt das Promotionsstipendium viel Raum für Selbstorganisation, erfordert gleichzeitig aber auch viel Selbstdisziplin. In jedem Falle sind regelmäßige Treffen mit einer Gruppe von Gleichgesinnten zu empfehlen, die nicht nur psychische Unterstützung in schwierigen Situationen gewährt, sondern auch eine externalisierte Kontrollfunktion erfüllen kann. Keinesfalls sollte die Doktorandin glauben, alles allein lösen zu müssen.

Wie nun entscheiden zwischen den beiden reizvollen Alternativen? Nach meiner - subjektiven - Einschätzung gibt es gerade für Juristinnen einen überaus triftigen Grund, allem Hype um den LL.M. zum Trotz, den Doktortitel zu bevorzugen - der Doktor schlägt sich in der Anrede nieder. Beabsichtigt eine Juristin in einer überwiegend von Männern bevölkerten Kanzlei, einem Wirtschaftsunternehmen oder einer anderen Männerdomäne zu arbeiten, empfiehlt sich im Namen ein Unterscheidungskriterium zu weiblichen Schreibkräften. Der LL.M. wird zwar als hübsches Anhängsel dem Namen nachgestellt, führt aber nicht zur Anrede „Magistra“. Auch im Staatsdienst erscheint der LL.M. weder auf dem Türschild noch in Urteilen. Viele Frauen in der Wirtschaft, in Großkanzleien und im Staatsdienst weisen deshalb auf die subtile Kraft des Doktortitels hin. Mir selbst wurde während des Referendariats von Anwälten mehrerer Großkanzleien nahegelegt, doch erst einen „Doktor“ zu machen, bevor ich als Frau ernsthaft Berücksichtigung finden könne. Auf Nachfrage wurde mir zum Selbstschutz geraten: „Sonst werden Sie nicht für voll genommen. “ Zeitaufwendig, aber gleichwohl immer verbreiteter ist es, beide Titel zu erwerben und von den positiven Erfahrungen beider Alternativen zu profitieren.

\section{Now, now! - Ein Erfahrungsbericht über ein Jahr in Südafrika und ein LL.M.-Programm}

\author{
Anne Brozat, LL.M. (UWC) \\ Promotionstipendiatin des Nachwuchsforscherkollegs „Compli- \\ ance“, Ruhr-Universität Bochum
}

So, endlich angekommen. Februar 2009 und ich stand am International Airport Cape Town. Das Gepäck fest im Griff, die Handtasche ebenfalls - in Südafrika kann man ja nie wissen. Kurz darauf saß ich im Auto und fuhr Richtung Kapstadt dem Tafelberg entgegen. Vor ein paar Stunden war ich aus dem kalten Deutschland aufgebrochen und nun im Sommer angekom- men. Ich freute mich auf das, was vor mir lag und auf dieses fremde spannende Land.

Begonnen hatte alles Ende August 2008 mit einer Anzeige in der NJW, in der die Humboldt Universität Berlin und die University of the Western Cape zu Bewerbungen für das neue englischsprachige LL.M.-Programm „Transnational Criminal Justice and Crime Prevention“, gefördert vom DAAD, aufriefen. Die Beschreibung des Programms klang spannend und passte genau zu meinem Interessengebiet. Es sollten Kurse zum Völkerstrafrecht und Wirtschaftsstrafrecht stattfinden, 